\title{
Acute thrombocytopenia: An uncommon complication occurring following transarterial chemoembolization in a patient with neuroendocrine hepatic metastases
}

\author{
PINGKUN XIE ${ }^{1}$ and ZHENG YUAN ${ }^{2,3}$ \\ ${ }^{1}$ Department of Radiology, Ningbo Beilun People's Hospital, Ningbo, Zhejiang 315800; \\ ${ }^{2}$ Department of Radiology, Shanghai 85 Hospital, Shanghai 200052; ${ }^{3}$ Department of Radiology, \\ Fudan University Shanghai Cancer Center, Shanghai 200032, P.R. China
}

Received July 17, 2014; Accepted April 30, 2015

DOI: $10.3892 / \mathrm{ol} .2015 .3232$

\begin{abstract}
Neuroendocrine tumors are a group of carcinomas that secrete various polypeptides with hormonal activity. A significant percentage of patients already have hepatic metastases at the time of initial diagnosis, and $80-90 \%$ of these tumors are inoperable at the time of presentation. Transarterial chemoembolization (TACE) is the preferred approach for the management of neuroendocrine hepatic metastases. Although the technique is relatively safe, it is associated with several complications. The present study reported the case of a patient with neuroendocrine hepatic metastases who developed acute thrombocytopenia following TACE. To the best of our knowledge, acute thrombocytopenia occurring after TACE in a patient with neuroendocrine hepatic metastases has not been previously reported. In the present study, the hypothetical etiopathogenetic mechanisms were also discussed.
\end{abstract}

\section{Introduction}

Neuroendocrine tumors (NETs) are a group of carcinomas that secrete various polypeptides with hormonal activity (1). These tumors arise from the amine precursor uptake and decarboxylation system, and have been identified in the gastrointestinal tract (entrochromaffin cells), the lung (Kulchitsky's cells, bronchus) and the pancreas (islet cells) (1-3). NETs are slowly growing tumors with an indolent course. A significant percentage of patients already have hepatic metastases at the time of initial diagnosis, and $80-90 \%$ of these tumors are inoperable at the time of presentation $(4,5)$. The presence of distant metastases is associated with a poor prognosis for

Correspondence to: Dr Zheng Yuan, Department of Radiology, Shanghai 85 Hospital, 1328 Huashan Road, Shanghai 200052, P.R. China

E-mail: yuanzheng0404@163.com

Key words: neuroendocrine, liver, metastases, transarterial chemoembolization neuroendocrine tumors: patients with carcinoid NETs have a 5 -year survival rate of only $22 \%$ (6). Liver metastases from NETs are invariably hypervascular, with a blood supply primarily from the hepatic artery. This feature, combined with the fact that the liver has a dual vascular supply, provides a good rationale for the use of hepatic transcatheter arterial embolization (TAE) to treat these metastases by inducing tumor ischemia. For higher metastatic load, TAE or chemoembolization (TACE) is the preferred approach for the management of symptomatic and local tumor control, as well as for improvement of the survival rate (7). Following a study by Moertel et al (8), which demonstrated a higher disease regression rate and a longer duration of regression with systemic chemotherapy following hepatic arterial occlusion than with occlusion alone, TACE (which used chemotherapy in addition to the embolic material) has often been favored over TAE. There is no consensus on the most effective chemotherapeutic agent for use in this procedure. Various chemotherapeutic agents, including doxorubicin, streptozocin, 5-fluorouracil, mitomycin C, cisplatin, and a combination of these agents have been used to perform TACE for hepatic metastases of NETs (9). However, there were no significant differences in the response rate to these agents (9). Complications of TACE are moderate in the majority of patients, with post-embolization syndrome being common. The present study reported the case of a patient with neuroendocrine hepatic metastases who developed acute thrombocytopenia following TACE, and discussed the hypothetical etiopathogenetic mechanisms involved.

\section{Case report}

A 56-year-old male, who had undergone surgical resection of a NET of the pancreas 17 months earlier, was admitted to the Fudan University Shanghai Cancer Center (Shanghai, China) for the treatment of multiple liver metastases from NET. Written informed consent was obtained from the patient for participation in the present study. The liver lesions were first observed 16 months after surgery. TACE was scheduled 1 month after the initial diagnosis was confirmed by histopathological examination of a needle biopsy. TACE was 
performed according to the following protocol: a selective 5-F catheter was introduced, and visceral angiography was carried out to assess the arterial blood supply to the liver and to confirm patency of the portal vein. Selective proper hepatic angiography revealed innumerable hypervascular tumors located throughout the liver. In order to treat lesions in the whole liver, the right and left hepatic arteries were separately catheterized using a coaxial technique and microcatheters (2.8 F; Terumo Corporation, Tokyo, Japan). Hepatic artery infusion chemotherapy was performed using cisplatin $60 \mathrm{mg}$ and floxuridine 1,000 $\mathrm{mg}$ (both from Zhejiang Hisun Pharmaceutical, Taizhou, China). Next, chemoembolization was performed using drugs mixed at a 2:1 ratio of epirubicin (60 mg; Zhejiang Hisun Pharmaceutical) and non-ionic contrast material (5 ml; Ultravist; Schering, Berlin, Germany) to iodized oil ( $8 \mathrm{ml}$; Lipiodol Ultra Fluide; Laboratoires Guerbet, Paris, France). No adverse events were noted during the interventional therapy.

Full blood counts were within normal ranges before the patient's treatment: hemoglobin, $159 \mathrm{~g} / 1$; white blood count

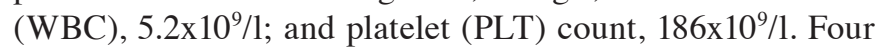
days after administering chemoembolization, hematological examination revealed the following results: hemoglobin,

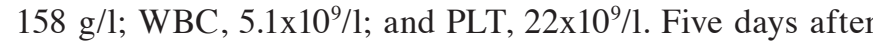
administering chemoembolization, the PLT count was $7 \times 10^{9} / 1$. Since grade 4 thrombocytopenia was diagnosed, platelet transfusions were administered. However, the PLT counts remained at levels below $10 \times 10^{9} / 1$ in spite of a total platelet transfusion of 20 units. Anti-nuclear antibody was negative, and no evidence of disseminated intravascular coagulopathy was observed. The platelet-associated immunoglobulin- $\mathrm{G}$ (IgG) level was $734 \mathrm{ng} / \mathrm{dl}$. A clinical diagnosis of autoimmune thrombocytopenia was made. Therapy with $40 \mathrm{mg} /$ day dexamethasone and $20 \mathrm{~g} /$ day immunoglobulin for five days were started on the seventh day after performing chemoembolization. The number of platelets increased immediately, with the PLT count being $51 \times 10^{9} / 1$ and $85 \times 10^{9} / 1$ on the second and third days after starting the steroid. Subsequent PLT counts remained stable at levels over 150x10 $/ 1$ (Fig. 1).

\section{Discussion}

The complications observed with TACE and TAE in the treatment of neuroendocrine hepatic metastases vary in the literature. Associated problems include liver abscesses, transient hepatorenal failure, pleural effusion, sepsis, bowel ischemia requiring surgery, septicemia requiring antibiotic therapy and hepatic infarction. Post-embolization syndrome is a further less severe adverse reaction, and is observed in the majority of patients. It may also cause a fever that subsides within a few days, leukocytosis, abdominal pain that may require morphine, and a transient increase in liver enzymes, particularly transaminases and lactate dehydrogenase, which tend to return to normal within a few days to 2-3 weeks. Increased bilirubin levels have also been noted. Ischemia of the biliary tree has been reported occasionally following embolization. In addition, elevation of alkaline phosphatase has been observed. The most appropriate means of reducing post-embolization syndrome is by keeping the patient well hydrated and in supportive care (10).

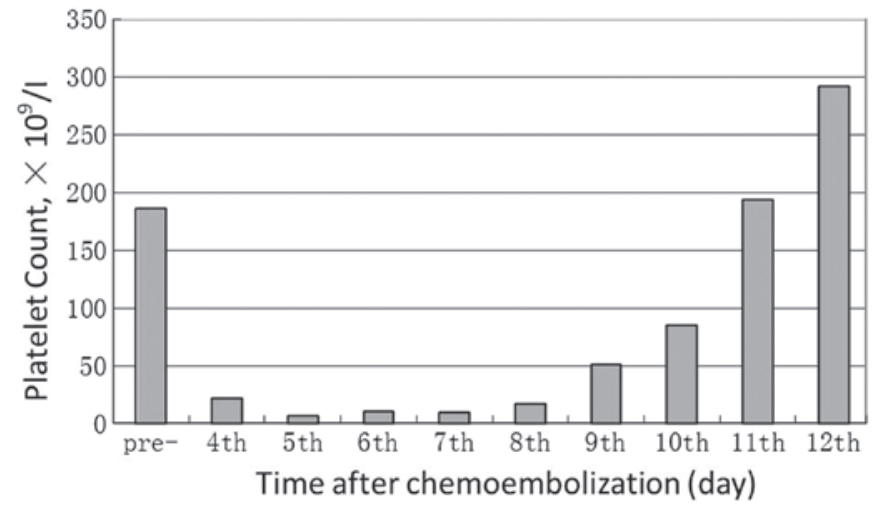

Figure 1. Temporal correlation between development of immune thrombocytopenia and transarterial chemoembolization.

In the case reported in the present study, four days after administering chemoembolization, hematological examination revealed an abnormal PLT count $\left(22 \times 10^{9} / 1\right)$. Thrombocytopenia was initially believed to have been caused by prolonged myelosuppression induced by chemotherapy. However, despite extensive repeated transfusions, thrombocytopenia was not improved, and there was no leukopenia during chemotherapy. It is unlikely that other factors may have caused thrombocytopenia. The diagnosis of immune thrombocytopenia was made based on the results of the elevated platelet-associated $\mathrm{IgG}$ level and the response to dexamethasone and immunoglobulin therapy.

Immune-mediated thrombocytopenia resembling idiopathic thrombocytopenic purpura accompanied by additional morbidities is known as secondary autoimmune thrombocytopenia (11). Common causes of thrombocytopenia in solid tumors are myelosuppression secondary to chemotherapy, bone marrow infiltration with malignant cells and increased peripheral utilization or destruction of platelets due to disseminated intravascular coagulation. Immune-mediated platelet destruction as a cause of thrombocytopenic syndromes in patients with solid tumors was first described by Kim et al (12). However, no antibody or kinetic verification tests were performed in this study. Schwartz et al (13) reported on eight patients with solid tumors having immune-mediated thrombocytopenia, and revealed evidence of antibodies attached to autologous platelets by radioimmune and release antibody assays. Further studies reveal that the frequency of accompanying solid tumors in patients with autoimmune thrombocytopenia is 5.6-12.9\% (12,13). Autoimmune thrombocytopenia has been observed in a number of solid tumors, including breast, lung, prostate, testis, kidney, skin and ovarian cancers. However, elevated platelet-associated IgG levels may also be induced by drugs (14). Therefore, in the case presented here, it was concluded that the elevated platelet-associated IgG may have been associated not only with tumors but also with drugs.

In conclusion, we have presented a case of immune thrombocytopenia in a patient with neuroendocrine hepatic metastases, which developed following TACE, and which was difficult to differentiate from thrombocytopenia caused by myelosuppression. We recommend that immune thrombocytopenia is considered in the differential diagnosis of thrombocytopenia in cancer patients. 


\section{Acknowledgements}

This study was supported by grants from the Science Technology Commission of Shanghai Municipality (nos. 0952nm03400, $11 \mathrm{~nm} 0504000$ and 124119a0100) and the National Natural Science Foundation of China (nos. 81301218 and 81301262).

\section{References}

1. Loewe C, Schindl M and Cejna M: Permanent transarterial embolization of neuroendocrine metastases of the liver using cyanoacrylate and lipiodol: assessment of mid- and long-term results. AJR Am J Roentgenol 180: 1379-1384, 2003.

2. Venook AP: Embolization and chemoembolization therapy for neuroendocrine tumors. Curr Opin Oncol 11: 39-44, 1999.

3. Kress O, Wagner HJ, Wied M, et al: Transarterial chemoembolization of advanced liver metastases of neuroendocrine tumors: a retrospective single centre analysis. Digestion 68: 94-101, 2003.

4. Proye C: Natural history of liver metastasis of gastroenteropancreatic neuroendocrine tumors: place for chemoembolization. World J Surg 25: 685-688, 2001.

5. Blonski WC, Reddy KR, Shaked A, et al: Liver transplantation for metastatic neuroendocrine tumor: a case report and a review of literature. World J Gastroenterol 11: 7676-7683, 2005.
6. Modlin IM and Sandor A: An analysis of 8305 cases of carcinoid tumors. Cancer 79: 813-829, 1997.

7. Vogl TJ, Naguib NN, Zangos S, et al: Liver metastases of neuroendocrine carcinomas: interventional treatment via transarterial embolization, chemoembolization and thermal ablation. Eur J Radiol 72: 517-528, 2009.

8. Moertel CG, Johnson CM, McKusick MA, et al: The management of patients with advanced carcinoid tumors and islet cell carcinomas. Ann Intern Med 120: 302-309, 1994.

9. Madoff DC, Gupta S, Ahrar K, Murthy R and Yao JC: Update on the management of neuroendocrine hepatic metastases. J Vasc Interv Radiol 17: 1235-1249, quiz 1250, 2006.

10. Touzios JG, Kiely JM, Pitt SC, et al: Neuroendocrine hepatic metastases: does aggressive management improve survival. Ann Surg 241: 776-783, 2005.

11. Bussel JB: Autoimmune thrombocytopenic purpura. Hematol Oncol Clin N Am 4: 179-191, 1990.

12. Kim HD and Boggs DR: A syndrome resembling idiopathic thrombocytopenic purpura in 10 patients with diverse forms of cancer. Am J Med 67: 371-377, 1979.

13. Schwartz KA, Slichter SJ and Harker LA: Immune-mediated platelet destruction and thrombocytopenia in patients with solid tumors. Br J Haematol 51: 17-24, 1982.

14. Kelton JG, Meltzer D, Moore J, et al: Drug induced thrombocytopenia is associated with increased binding of $\mathrm{IgG}$ to platelets both in vivo and in vitro. Blood 58: 524-529, 1981. 